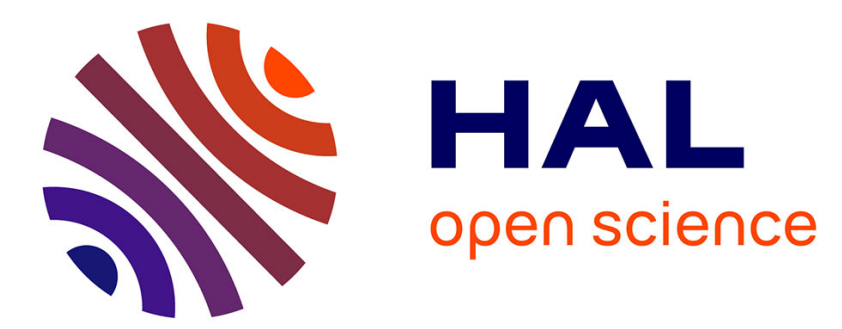

\title{
The adjoint method for general EEG and MEG sensor-based lead field equations
}

Théodore Papadopoulo, Sylvain Vallaghé, Maureen Clerc

\section{To cite this version:}

Théodore Papadopoulo, Sylvain Vallaghé, Maureen Clerc. The adjoint method for general EEG and MEG sensor-based lead field equations. Biomag 2010 - 17th International Conference on Biomagnetism, S. Supek, Mar 2010, Dubrovnik, Croatia. pp.105-108, 10.1007/978-3-642-12197-5_20 . inria-00497088

\section{HAL Id: inria-00497088 \\ https://hal.inria.fr/inria-00497088}

Submitted on 2 Jul 2010

HAL is a multi-disciplinary open access archive for the deposit and dissemination of scientific research documents, whether they are published or not. The documents may come from teaching and research institutions in France or abroad, or from public or private research centers.
L'archive ouverte pluridisciplinaire HAL, est destinée au dépôt et à la diffusion de documents scientifiques de niveau recherche, publiés ou non, émanant des établissements d'enseignement et de recherche français ou étrangers, des laboratoires publics ou privés. 


\title{
The adjoint method for general EEG and MEG sensor-based lead field equations
}

\author{
Théodore Papadopoulo ${ }^{1}$, Sylvain Vallaghé ${ }^{1}$ and Maureen Clerc ${ }^{1}$ \\ ${ }^{1}$ Odyssée Project-Team, INRIA Sophia Antipolis, France
}

\begin{abstract}
The inverse source problem in electroencephalography (EEG) and magnetoencephalography (MEG) generally uses a lead field, which relates any source in the brain to its measurements at the sensors. For complex geometries, there is no analytical formula of the lead field. The common approach is to numerically compute the value of the lead field for a finite number of point sources (dipoles). There are several drawbacks: the model of the source space is fixed (a set of dipoles) and the computation can be expensive for as much as 10000 dipoles. The common idea to bypass these problems is to compute the lead field from a sensor point of view. We show how the adjoint method can be used to derive general EEG and MEG sensorbased lead field equations. Within a simple framework, we provide a complete review of the explicit lead field equations, and we are able to extend these equations to non-point like sensors.
\end{abstract}

Keywords - Electroencephalography, Magnetoencephalography, Forward problem, Adjoint method.

\section{INTRODUCTION}

Electroencephalography (EEG) and magnetoencephalography (MEG) inverse problems traditionally optimizes a source configuration by "comparing" actual measurements to quantities simulated using a forward model that predicts the electric potential or magnetic field. The EEG forward problem is governed by the following partial differential equation:

$$
\left\{\begin{array}{lll}
\nabla \cdot(\sigma \nabla V) & =\nabla \cdot \mathbf{J}^{\mathrm{p}} & \text { in } \Omega \\
\sigma \nabla V \cdot \mathbf{n} & =0 & \text { on } \partial \Omega
\end{array}\right.
$$

where $V$ is the electric potential, $\sigma$ is the conductivity, $\mathbf{J}^{\mathrm{p}}$ is the primary current vector representing brain electrical activity. $\Omega$ represents the head domain (an open bounded region of the ambient space $\mathbb{R}^{3}$ ). Similarly, the magnetic field is given by the Biot-Savart law:

$$
\mathbf{B}\left(\mathbf{r}_{i}\right)=\mathbf{B}_{0}\left(\mathbf{r}_{i}\right)-\frac{\mu_{0}}{4 \pi} \int_{\Omega} \sigma \nabla V \times \nabla\left(\frac{1}{R}\right) d r,
$$

with $\mathbf{B}_{0}\left(\mathbf{r}_{i}\right)=\frac{\mu_{0}}{4 \pi} \int_{\Omega} \mathbf{J}^{\mathrm{p}} \times \nabla\left(\frac{1}{R}\right) d r$ and $R=\left\|\mathbf{r}_{i}-\mathbf{r}\right\|$. It is obvious that both $V$ and $\mathbf{B}$ are linear functions of $\mathbf{J}^{\mathrm{p}}$. Because of this property, the predictions of the forward problem at the sensor locations (the only information used by inverse problems) can be conveniently summarized by a leadfield, which is a linear operator $\mathbf{L}$ that maps source activations to simulated potentials of magnetic fields at sensor locations. Since it is linear, this operator can be written using the Riesz representation theorem:

$$
\mathbf{L}\left(\mathbf{J}^{\mathrm{p}}\right)=<\frac{\partial \mathbf{L}}{\partial \mathbf{J}^{\mathrm{p}}}, \mathbf{J}^{\mathrm{p}}>
$$

where $<,>$ denotes for the inner product ${ }^{1}$ of functions in $\mathbb{R}^{3}$ :

$$
<u, v>=\int_{\Omega} u(\mathbf{r}) \cdot v(\mathbf{r}) d r .
$$

Traditionally, the forward problem equations are discretized and the leadfield is represented by the matrix $\frac{\partial \mathbf{L}}{\partial \mathbf{J}^{\mathrm{p}}}$ whose number of lines equals the number of sensors and whose number of columns equals the number of parameters of the dipoles considered as possible sources (around 10000 in distributed source models). Each column of this matrix gives the values of the electric or magnetic measurements at the sensor locations for a given dipole parameter. A simple way to compute the leadfield matrix is thus to solve as many forward problems as there are source parameters, which can be quite time consuming with detailed realistic geometries for the head. One solution to bypass this problem relies on some matrix manipulations on the discretized problem so as to estimate rows of the leadfield matrix (instead of columns) [1,2]. This is quite interesting as there are generally much less sensors than source positions and as the resulting computation is very similar to the original forward problem. Another solution is to work in the continuous domain, and to use the Helmholtz reciprocity principle to express the leadfields as the solutions of different forward problems [1, 3, 4].

Both these approaches can been explained using the adjoint method [5], which is applied here in the continuous domain to both the EEG and MEG leadfields. As the approach is very general, it is quite easy to include within this framework new information such as sensor geometry.

\section{THE ADJOINT METHOD}

Let $p$ represent a parameter which can be a real scalar or vector function. For any $p$, we consider the (hopefully

\footnotetext{
${ }^{1}$ For scalar functions the inner product inside the integral becomes a simple product.
} 
unique) function $v$ which satisfies the state equation:

$$
\mathbf{A} v=b(p),
$$

where $\mathbf{A}$ is a linear operator of $\mathbb{R}^{3}$ and $b$ is a differentiable function of $\mathbb{R}^{3}$. To give some intuition, in our case the state function $v$ is the electric potential $V(\mathbf{r})$, the parameter $p$ is the primary source current vector $\mathbf{J}^{\mathrm{p}}(\mathbf{r})$, the operator $\mathbf{A}$ is the generalized Laplacian $\nabla \cdot(\sigma \nabla$. $)$ and $b$ is the divergence operator.

Let us consider a "measurement function" $g$ which is a linear functional of $p$ and $v$. From the Riesz representation theorem, there exists $m$ and $n \in E$ such that $g(p, v)=\langle m, v\rangle$ $+<n, p\rangle$. To compute the differential of $g$ with respect to $p$ (knowing that $v$ also depends linearly on $p$ ), we first build a Lagrangian $\mathscr{L}$ by adding to the measurement equation the inner product of the state equation with a Lagrange multiplier $w$ :

$$
\mathscr{L}(p, v, w)=<m, v>+<n, p>+<w, \mathbf{A} v-b(p)>.
$$

Notice that $g(p, v(p))=\mathscr{L}(p, v(p), w)$ for all $w$ as $\mathbf{A} v(p)=$ $b(p)$. Assuming that $\mathscr{L}$ is differentiable with respect to both $v$ and $p$, the Lagrange multiplier $w$ is chosen such that $\frac{\partial \mathscr{L}}{\partial v}(p, v(p), w) \boldsymbol{\delta} v=<m, \boldsymbol{\delta} v>+<w, \mathbf{A} \boldsymbol{\delta} v>=$ 0 for all $\delta v$. Introducing the adjoint operator $\mathbf{H}^{*}$ of a linear operator $\mathbf{H}$, such that $\langle x, \mathbf{H} y\rangle=\left\langle\mathbf{H}^{*} x, y\right\rangle$, the previous equation can be rewritten as $\left\langle m+\mathbf{A}^{*} w, \delta v\right\rangle=0$ for all $\delta v$, which is equivalent to:

$$
\mathbf{A}^{*} w=-m .
$$

This equation is called the adjoint equation. With this choice for $w$, the differential of $g$ can be written as:

$$
\delta g=\frac{\partial \mathscr{L}}{\partial p}(p, v(p), w) \delta p=<n-\frac{\partial b^{*}}{\partial p} w, \delta p>.
$$

Since $g$ is linear in $p, g(p)=<\frac{\partial g}{\partial p}, p>\cdot g(p)$ being a measurement, $\frac{\partial g}{\partial p}$ is exactly the leadfield $\mathbf{L}$ and Eq. (6) exactly expresses this operator as a function of the adjoint variable $w:$

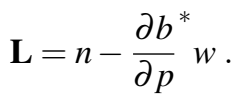

\section{APPLICATION TO M/EEG LEADFIELDS}

This section briefly details the application of the previous result to M/EEG leadfields. For more detailed calculations, the reader is referred to [6]. Using the previous result first requires the computation of the adjoint operators associated to our state equation (1) (i.e. $\mathbf{A}^{*}$ and $\frac{\partial b}{\partial p}^{*}$ ). In our case, $v$ is the potential $V(\mathbf{r}), p$ is the primary source $\mathbf{J}^{\mathrm{p}}(\mathbf{r})$ and $b$ is the divergence operator, which is linear so that $\frac{\partial \nabla \cdot \mathbf{J}^{\mathrm{p}}}{\partial \mathbf{J}^{\mathrm{p}}} \mathbf{J}^{\mathrm{p}}=\nabla \cdot \mathbf{J}^{\mathrm{p}}$. Using the divergence theorem:

$\left.\left\langle w, \nabla \cdot \mathbf{J}^{\mathrm{p}}\right\rangle=\int_{\Omega} w \nabla \cdot \mathbf{J}^{\mathrm{p}} d r=-\int_{\Omega} \mathbf{J}^{\mathrm{p}} \cdot \nabla w d r=<-\nabla w, \mathbf{J}^{\mathrm{p}}\right\rangle$,

because sources are restricted to be strictly inside the head, so the boundary integrals vanish. Thus:

$$
\frac{\partial b^{*}}{\partial p} w=\frac{\partial \nabla \cdot \mathbf{J}^{\mathrm{p}}}{\partial \mathbf{J}^{\mathrm{p}}} w=-\nabla w .
$$

For $\mathbf{A}^{*}$, we start with $\langle w, \mathbf{A} v>$ :

$$
<w, \mathbf{A} v>=\int_{\Omega} w \nabla \cdot(\sigma \nabla V) d r .
$$

Assuming that $w$ is continuous on $\Omega$, but that $\sigma \nabla w \cdot \mathbf{n}$ can be discontinuous at tissue interfaces, the divergence theorem applied twice leads to (using the facts that $\sigma \nabla V \cdot \mathbf{n}=0$ on $\partial \Omega$ and that $V$ and $\sigma \nabla V \cdot \mathbf{n}$ are continuous functions over $\Omega$ ):

$$
\begin{aligned}
<A^{*} w, v> & =\int_{\Omega} w \nabla \cdot(\sigma \nabla V) d r \\
& =\int_{\Omega} V \nabla \cdot(\sigma \nabla w) d r-\sum_{k} \int_{S_{k}} V[\sigma \nabla w \cdot \mathbf{n}]_{S_{k}} d s,
\end{aligned}
$$

where $S_{k}$ are the tissue interfaces and $[.]_{S_{k}}$ denotes the jump of a function on a given surface $S_{k}$.

\section{A. EEG leadfield}

One line of the EEG leadfield is the potential of an electrode located at point $\mathbf{r}_{i}$ with respect to some reference electrode located at $\mathbf{r}_{0}$ (mean reference can also easily be handled with slightly more complicated formulae). Computing a leadfield for dipoles with fixed orientations can also be done easily following the same principle.

$$
\begin{aligned}
g_{E E G}(p, v) & =g_{E E G}\left(\mathbf{J}^{\mathrm{p}}, V\right)=V\left(\mathbf{r}_{i}\right)-V\left(\mathbf{r}_{0}\right) \\
& =\int_{\partial \Omega} V(\mathbf{r})\left(\boldsymbol{\delta}\left(\mathbf{r}_{i}\right)-\delta\left(\mathbf{r}_{0}\right)\right) d r,
\end{aligned}
$$

where the notation $\delta(\mathbf{r})$ refers to the Dirac distribution at $\mathbf{r} \in$ $\partial \Omega$. The integral is restricted to $\partial \Omega$ since all the EEG sensors are located on this surface. As $g\left(\mathbf{J}^{\mathrm{p}}, V\right)$ does not depend on $\mathbf{J}^{\mathrm{p}}$, this leads to $m_{E E G}=\delta\left(\mathbf{r}_{i}\right)-\delta\left(\mathbf{r}_{0}\right)$ and $n_{E E G}=0$. Equating all the surfacic and volumic terms in the linear forms shows that $\left[\sigma_{k} \nabla w \cdot \mathbf{n}\right]_{S_{k}}=0$ for all inner interfaces (as there are no 
other surfacic terms for those), hence only the last surface $(\partial \Omega)$ is to be kept in the sum in Eq. (9):

$$
<\mathbf{A}^{*} w, V>=\int_{\Omega} V \nabla \cdot(\sigma \nabla w) d r-\int_{\partial \Omega} V[\sigma \nabla w \cdot \mathbf{n}]_{\partial \Omega} d s .
$$

From Eq. (5), $w$ satisfies:

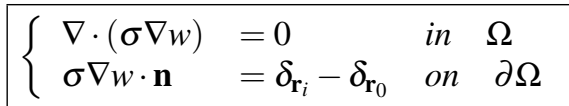

Combining Eq. (7) and Eq. (8), the EEG leadfield for punctual electrodes is:

$$
\mathbf{L}_{E E G}=\nabla w
$$

\section{B. MEG leadfield}

For MEG, the only difference is in the type of measurements: $g_{M E G}(p, v)=g_{M E G}\left(\mathbf{J}^{\mathrm{p}}, V\right)=\mathbf{B}\left(\mathbf{r}_{i}\right) \cdot \mathbf{d}_{i}$, with $\mathbf{B}$ given by the Biot-Savart law (2). This law has two terms, $\mathbf{B}_{0}$ depending on $\mathbf{J}^{\mathrm{p}}$ and the other term depending on $V$. Applying the adjoint state approach requires the computation of the corresponding $m_{M E G}$ and $n_{M E G}$.

$n_{M E G}$ corresponds to the $\mathbf{B}_{0}$ term:

$$
\begin{aligned}
\mathbf{B}_{0}\left(\mathbf{r}_{i}\right) \cdot \mathbf{d}_{i} & =\frac{\mu_{0}}{4 \pi} \int_{\Omega}\left(\mathbf{J}^{\mathrm{p}} \times \nabla\left(\frac{1}{R}\right)\right) \cdot \mathbf{d}_{i} d r \\
& =\frac{\mu_{0}}{4 \pi} \int_{\Omega} \mathbf{J}^{\mathrm{p}} \cdot\left(\nabla\left(\frac{1}{R}\right) \times \mathbf{d}_{i}\right) d r .
\end{aligned}
$$

Thus

$$
n_{M E G}=\frac{\mu_{0}}{4 \pi}\left(\nabla\left(\frac{1}{R}\right) \times \mathbf{d}_{i}\right) .
$$

$m_{M E G}$ corresponds to the Ohmic current term:

$$
\begin{aligned}
<m_{M E G}, \mathbf{J}^{\mathrm{p}}>= & -\frac{\mu_{0}}{4 \pi} \int_{\Omega} \sigma \nabla V \cdot\left(\nabla\left(\frac{1}{R}\right) \times \mathbf{d}_{i}\right) d r \\
= & \frac{\mu_{0}}{4 \pi} \int_{\Omega} V \nabla \cdot \sigma\left(\left(\frac{1}{R}\right) \times \mathbf{d}_{i}\right) d r- \\
& \frac{\mu_{0}}{4 \pi} \int_{\Omega} \nabla \cdot V \sigma\left(\left(\frac{1}{R}\right) \times \mathbf{d}_{i}\right) d r \\
= & \frac{\mu_{0}}{4 \pi} \int_{\Omega} V \nabla \cdot \sigma\left(\left(\frac{1}{R}\right) \times \mathbf{d}_{i}\right) d r- \\
& \frac{\mu_{0}}{4 \pi} \sum_{k} \int_{S_{k}} V[\sigma]_{S_{k}}\left(\left(\frac{1}{R}\right) \times \mathbf{d}_{i}\right) \cdot \mathbf{n} d s .
\end{aligned}
$$

Thus $m_{M E G}$ has both a volumic term and several surfacic terms. Equating all the surfacic and volumic terms in the linear forms yields the following equation for the adjoint:

$$
\left\{\begin{array}{lll}
\nabla \cdot(\sigma \nabla w) & =-\frac{\mu_{0}}{4 \pi} \nabla \cdot\left(\sigma \nabla\left(\frac{1}{R}\right) \times \mathbf{d}_{i}\right) & \text { in } \Omega_{k}, \\
{[\sigma \nabla w \cdot \mathbf{n}]_{S_{k}}} & =-\frac{\mu_{0}}{4 \pi}[\sigma]_{S_{k}} \nabla\left(\frac{1}{R}\right) \times \mathbf{d}_{i} \cdot \mathbf{n} & \text { on } S_{k}
\end{array}\right.
$$

Note that if the conductivity $\sigma$ is constant and isotropic in a domain $\Omega_{k}$, then $\nabla \cdot\left(\sigma \nabla\left(\frac{1}{R}\right) \times \mathbf{d}_{i}\right)=0$ and $w$ is harmonic in each domain $\Omega_{k}$. This result has been used previously for the numerical computation of the MEG leadfield $[3,4]$.

Combining Eq. (7), Eq.(12) and Eq. (8), the MEG leadfield for a punctual magnetometer is:

$$
\mathbf{L}_{M E G}=\nabla w+\frac{\mu_{0}}{4 \pi}\left(\nabla\left(\frac{1}{R}\right) \times \mathbf{d}_{i}\right) .
$$

\section{INCORPORATING SENSOR GEOMETRY}

\section{A. MEG: Squids geometry}

A magnetometer measures the flux of the magnetic field through a small loop. For a magnetometer $i$, let $M_{i}$ be the surface enclosed by the loop, and $\mathbf{d}_{i}$ the unitary vector normal to $M_{i}$. The function $g$ can thus be written as $g\left(\mathbf{J}^{\mathrm{p}}\right)=\int_{M_{i}} \mathbf{d}_{i}$. $\mathbf{B}\left(\mathbf{r}^{\prime}\right) d r^{\prime}$. Switching the integrals over $M_{i}$ and $\Omega$ and since the only dependence of $\mathbf{B}\left(\mathbf{r}^{\prime}\right)$ on $\mathbf{r}^{\prime}$ is in $R$, it is quite easy to show that following the lines of section III.B yields:

$$
\begin{aligned}
& \left\{\nabla \cdot(\sigma \nabla w)=-\frac{\mu_{0}}{4 \pi} \int_{M_{i}} \nabla \cdot\left(\sigma \nabla\left(\frac{1}{R}\right) \times \mathbf{d}_{i}\right) d r^{\prime} \quad \text { in } \Omega_{k},\right. \\
& \left\{[\sigma \nabla w \cdot \mathbf{n}]_{S_{k}}=-\frac{\mu_{0}}{4 \pi}[\sigma]_{S_{k}} \nabla\left(\frac{1}{R}\right) \times \mathbf{d}_{i} \cdot \mathbf{n} \quad \text { on } S_{k}\right.
\end{aligned}
$$

As in the point wise case, the term $\int_{M_{i}} \nabla \cdot\left(\sigma \nabla\left(\frac{1}{R}\right) \times \mathbf{d}_{i}\right) d r^{\prime}$ vanishes if $\sigma$ is constant and isotropic in a domain $\Omega_{k}$. The resulting expression for the leadfield is:

$$
\mathbf{L}_{M E G}=\nabla w+\frac{\mu_{0}}{4 \pi} \int_{M_{i}}\left(\nabla\left(\frac{1}{R}\right) \times \mathbf{d}_{i}\right) d \mathbf{r}^{\prime} .
$$

This formulation is the same as the one given in [2]. Gradiometers can be treated similarly. Generally, MEG manufacturers give a set of positions and weights for each sensor, and the linear combination of the magnetic field at these positions using these weights is meant to recreate the measurement. But this requires as many adjoint problems as there are positions. The main advantage of the proposed approach is to require a single adjoint problem per sensor, reducing notably the needed for the leadfield computation.

\section{B. EEG: Electrode surfaces}

Incorporating the surface of EEG electrodes in the model is more complicated as the electrode perturbs the electrical field due to the shunt effect: potential is constant at the surface of the electrodes due to the high conducting electrode (gel and metal). This effect has been modeled for EEG [7] and 
requires a modification of the boundary condition of the state equation (1), which becomes:

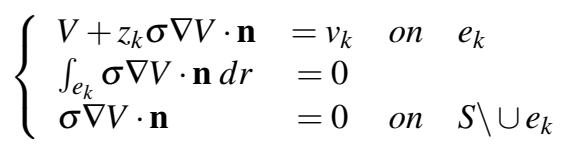

where $e_{k}$ is the $k$-th electrode, $v_{k}$ is the constant value of the potential on the $k$-th electrode and $z_{k}$ is the effective contact impedance which models the electrochemical effect at the skin-electrode interface. Following the adjoint approach yields the same leadfield equation as in the pointwise EEG case (Eq. (11)). However, the boundary condition of the adjoint state equation for $w$ is transformed into:

$$
\left\{\begin{array}{l}
w+z_{k} \sigma \nabla w \cdot \mathbf{n}=W_{k} \quad \text { on } e_{k}, \\
\int_{e_{k}} \sigma \nabla w \cdot \mathbf{n} d r= \begin{cases}1 & k=i \\
-1 & k=0 \\
0 & \text { otherwise }\end{cases} \\
\sigma \nabla w \cdot \mathbf{n}=0 \quad \text { on } \quad S \backslash \cup e_{k},
\end{array}\right.
$$

where $W_{k}$ is a constant.

\section{RESULTS}

To illustrate the approach, lead field for magnetometers are computed for a standard three nested spheres model with radii of $0.87,0.92,1$, meant to represent brain, skull and scalp tissues with respective conductivities of $1,0.02,1$. While it is known that in a spherical geometry the magnetic field outside the conductor does not depend on the conductivities, we intentionally put different conductivities in our model to test that the numerical solution is actually similar to the case of a homogeneous sphere. Computations were made using a finite element method presented in [8] and results were compared with the ground truth given by the analytical formulation. The magnetometer is oriented in the $x$ direction, positioned at a distance of 0.03 of the outermost sphere, and has a radius of 0.015. The differential equation of section IV A was solved, using our finite element method. The integral term over the magnetometer loop was computed after applying Stockes theorem with a Gauss-Kronod method using 61 points. Figure 1 shows the absolute error is similar and small for three dipole orientations. More results can be found in [6].

\section{CONCLUSION}

The adjoint method is a powerful general tool to derive efficient leadfield computation for various sensors types.

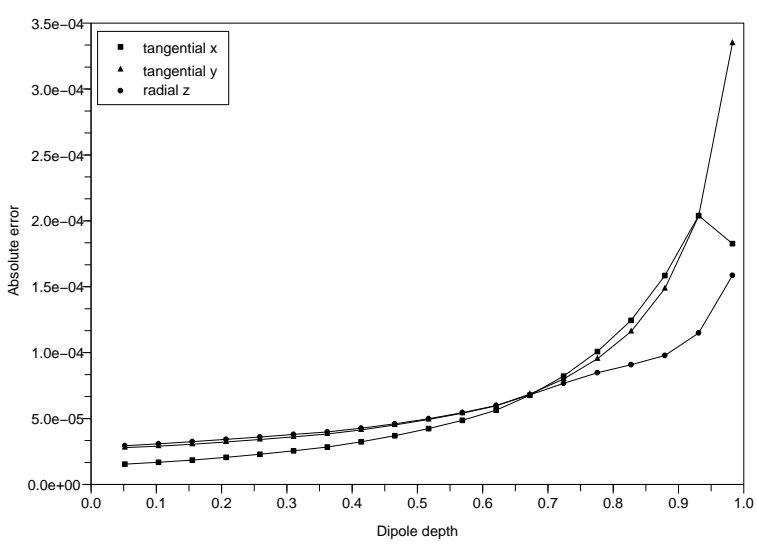

Fig. 1: Absolute error between numerical and analytical solution with respect to dipole depth.

\section{ACKNOWLEDGEMENTS}

This work is partly supported by contract EADS 2118.

\section{REFERENCES}

1. Weinstein D., Zhukov L., Johnson C.. Lead-field bases for electroencephalography source imaging Annals of Biomedical Engineering. 2000;28:1059-1164.

2. Wolters C. H., Grasedyck L., Hackbusch W.. Efficient computation of lead field bases and influence matrix for the FEM-based EEG and MEG inverse problem Inverse Problems. 2004;20:1099-1116.

3. Nolte G.. The magnetic lead field theorem in the quasi-static approximation and its use for magnetoencephalography forward calculation in realistic volume conductors Physics in Medicine and Biology. 2003;48:3637-3652.

4. Schimpf P. H.. Application of quasi-static magnetic reciprocity to finite element models of the MEG lead-field IEEE Transactions on Biomedical Engineering. 2007;54:2082-2088.

5. Lions J.L.. Optimal control of systems governed by partial differential equations. Springer 1971.

6. Vallaghé S., Papadopoulo T., Clerc M.. The adjoint method for general EEG and MEG sensor-based lead field equations Physics in Medicine and Biology. 2009;54:135-147.

7. Ollikainen Jorma O., Vauhkonen Marko, Karjalainen Pasi A., Kaipio Jari P.. Effects of electrode properties on EEG measurements and a related inverse problem Medical Engineering \& Physics. 2000;22:535545.

8. Papadopoulo Théodore, Vallaghé Sylvain. Implicit Meshing for Finite Element Methods using Levelsets in Proceedings of MMBIA 072007.

Author: Théo Papadopoulo Institute: INRIA Sophia-Antipolis

Street: 2004 route des Lucioles, BP 93

City: Sophia-Antipolis, Cedex

Country: France

Email: Theodore.Papadopoulo@sophia.inria.fr 